\section{(6) OPEN ACCESS}

\title{
Stability of mild cognitive impairment in newly diagnosed Parkinson's disease
}

\author{
Rachael A Lawson, ${ }^{1}$ Alison J Yarnall, ${ }^{1}$ Gordon W Duncan, ${ }^{1,2}$ David P Breen, ${ }^{3}$ \\ Tien K Khoo, ${ }^{4,5}$ Caroline H Williams-Gray, ${ }^{3}$ Roger A Barker, ${ }^{3}$ David J Burn, ${ }^{1}$ \\ On behalf of the ICICLE-PD study group
}

${ }^{1}$ Institute of Neuroscience, Newcastle University, Newcastle upon Tyne, UK

${ }^{2}$ Centre for Clinical Brain Sciences, University of Edinburgh, Edinburgh, UK JJohn van Geest Centre for Brain Repair, University of Cambridge, Cambridge, UK ${ }^{4}$ School of Medicine \& Menzies Health Institute Queensland, Griffith University, Southport, Queensland, Australia

${ }^{5}$ School of Medicine, University of Wollongong, Wollongong, New South Wales, Australia

Correspondence to Dr Rachael A Lawson, Clinical Ageing Research Unit, Institute of Neuroscience, Newcastle University Institute for Ageing, Newcastle University, Campus for Ageing and Vitality, Newcastle upon Tyne NE4 5PL, UK; rachael.lawson@ncl.ac.uk

Received 17 October 2016 Revised 12 December 2016 Accepted 14 December 2016 Published Online First 9 February 2017

\section{CrossMark}

To cite: Lawson RA Yarnall AJ, Duncan GW, et al. J Neurol Neurosurg Psychiatry 2017:88:648-652.

\section{ABSTRACT}

Background Mild cognitive impairment (MCI) is common in early Parkinson's disease (PD). We evaluated the stability of PD-MCl over time to determine its clinical utility as a marker of disease.

Methods 212 newly diagnosed participants with PD were recruited into a longitudinal study and reassessed after 18 and 36 months. Participants completed a range of clinical and neuropsychological assessments. PD-MCI was classified using Movement Disorders Society Task Force level I (Montreal Cognitive Assessment <26) and level II (using cut-offs of 1, 1.5 and 2SD) criteria.

Results After 36 months, $75 \%$ of participants returned; $8 \%$ of patients had developed a dementia all of which were previously PD-MCI. Applying level I criteria, $70 \%$ were cognitively stable, $19 \%$ cognitively declined and $11 \%$ improved over 36 months. Applying level II criteria (1, 1.5 and 2SD), 25\% were cognitively stable, $41 \%$ cognitively declined, 15\% improved and $19 \%$ fluctuated over 36 months. 18\% of participants reverted to normal cognition from PD-MCI.

Discussion Cognitive impairment in PD is complex, with some individuals' function fluctuating over time and some reverting to normal cognition. PD-MCl level I criteria may have greater clinical convenience, but more comprehensive level II criteria with 2SD cut-offs may offer greater diagnostic certainty.

\section{INTRODUCTION}

Cognitive impairment in Parkinson's disease (PD) is common, ultimately $80 \%$ of patients may develop dementia (PDD). ${ }^{1}$ Mild cognitive impairment in PD (PD-MCI) may be a prodromal stage of PDD. Guidelines to identify PD-MCI have been proposed by the Movement Disorder Society (MDS). ${ }^{2}$ The MDS criteria have yet to be validated, but several recent studies have investigated the number and optimal assessments, and best cut-offs to define PD-MCI. ${ }^{34}$

The MDS PD-MCI guidelines specify that in order to meet criteria for this diagnosis, a patient with PD must exhibit gradual cognitive decline (reported by the patient, informant or clinician) that is not severe enough to impair functional independence or activities of daily living. ${ }^{2}$ The patient should not have another primary explanation for their cognitive impairment such as delirium, stroke, major depressive disorder or head trauma. The patient should also not have any other PD-associated conditions that may significantly interfere with cognitive testing (eg, motor impairment, severe anxiety, daytime somnolence or psychosis).

Litvan et $a l^{2}$ classified PD-MCI criteria using either level I criteria (primarily for use in a clinical setting) or more stringent level II criteria (primarily for use in a research setting). Level I criteria require a less comprehensive battery of tests, for example, impairment on a global cognitive test which has been validated in PD such as the Montreal Cognitive Assessment (MoCA), or impairment on at least two tests in a limited battery of neuropsychological tests. Application of level II criteria requires at least two neuropsychological tests across each of five cognitive domains: attention, executive function, visuospatial function, memory and language. ${ }^{2}$ Patients meeting level II criteria should either be impaired in two or more tests in one cognitive domain (single domain PD-MCI) or in at least one test in two or more different domains (multidomain PD-MCI). Impairment is defined as performance of 1-2SD below appropriate norms, significant decline on serial neuropsychological testing, or a decline from premorbid levels. Level II criteria also include optional subtype classification: single domain or multidomain classification as well as type of impairment.

The longitudinal characteristics of PD-MCI are unknown, and whether it is a stable state, likely to decline or even improve over time. A multicentre study investigating the stability of MCI and dementias found that MCI was associated with diagnostic uncertainty. ${ }^{5}$ We hypothesised that PD-MCI may also be associated with prognostic uncertainty, which could be problematic in terms of clinical management, while causing unnecessary distress for patients. This study sought to determine the stability and clinical utility of PD-MCI in newly diagnosed patients with PD over 36 months.

\section{METHODS}

This study was approved by the Newcastle and North Tyneside 1 Research Ethics Committee. All participants provided written informed consent.

Recently diagnosed patients with PD were recruited from the community and outpatient clinics in Newcastle and Cambridgeshire, UK as part of the Incidence of Cognitive Impairment in Cohorts with Longitudinal Evaluation in PD (ICICLE-PD) study $^{67}$ and were evaluated at 18 -month intervals. At each assessment, demographic and clinical data 
were collected including MDS Unified Parkinson's Disease Rating Scale (MDS-UPDRS) Part III, Geriatric Depression Scale (GDS-15) and levodopa equivalent daily dose (LEDD). ${ }^{8}$

A detailed schedule of neuropsychological tests was performed at each assessment and has been described previously. ${ }^{6}$ Attention was assessed using Power of Attention (PoA) and Digit Vigilance Accuracy from the Cognitive Drug Research Battery. ${ }^{9}$ Executive function was assessed using the One Touch Tower of London (OTS) from the Cambridge Neuropsychological Test Automated Battery (CANTAB), ${ }^{10}$ phonemic fluency and semantic fluency. ${ }^{11}$ Memory was assessed using spatial and pattern recognition memory (SRM and PRM), and paired associate learning (PAL) subsets from the CANTAB. Visuospatial function was measured using modified scoring for copying interlocking pentagons. ${ }^{12}$ Language was measured using the naming and language scores from the MoCA. ${ }^{13}$

We used modified level II criteria to classify PD-MCI as our study design predated the MDS Task Force PD-MCI criteria (described by Yarnall et $\mathrm{al}^{6}$ ). We applied cut-offs of 1.0 ( $\geq 1$ but $<1.5 \mathrm{SD}), 1.5(\geq 1.5$ but $<2 \mathrm{SD})$ and $2.0(\geq 2 \mathrm{SD})$ SDs below normative values (controls), or an approximation to the normal distribution, to classify PD-MCI. We also applied level I PD-MCI criteria, with cognitive impairment defined as an MoCA score $<26 .{ }^{2}$ For both criteria, semistructured interviews were conducted with participants and their carers to determine subjective cognitive symptoms and functional independence. We classified participants according to the MDS criteria: single domain or multidomain PD-MCI and the domains impaired (described by Lawson $e^{a l}{ }^{14}$ ).

Data were examined for normality, and means were compared using analyses of variance or Kruskal-Wallis tests as appropriate (SPSS V.21.0). The $\chi^{2}$ tests were used to compare between-group distributions of proportion. Cochran's Q test compared between-group proportions over time.

\section{RESULTS}

Two hundred and twelve newly diagnosed participants with PD completed baseline assessments; $190(89.6 \%)$ and 158 (74.5\%) returned for 18-month and 36-month evaluations, respectively (mean of $3.1 \pm 0.2$ years). Demographic and clinical characteristics of participants at each time point are presented in table 1. Participants with cognitive impairment tended to be older, had completed fewer years of education, and had greater motor severity and lower global cognition scores $(\mathrm{p}<0.01$ for all).

Figure 1 shows the changes in cognitive classification between groups at each time point using level II (figure 1A) and level I (figure 1B) PD-MCI criteria. Between baseline and 18 months, $43 \%$ had stable cognition, 30\% cognitively declined and $15 \%$ improved in terms of their PD-MCI classification (1 vs 1.5 vs 2SD) using level II criteria. Between 18 and 36 months, 35\% of remaining participants had stable cognition, $29 \%$ cognitively declined and 18\% improved (figure 1A). Between baseline and 36 months, $27 \%(\mathrm{n}=58)$ were cognitively stable, $33 \%(\mathrm{n}=71)$ cognitively declined and 14\% ( $n=29)$ improved; $8 \%(n=18)$ developed PDD.

As shown in figure $1 \mathrm{~A}$, the majority of cognitively stable participants were classified as normal cognition (PD-CN), with $20 \%$ of participants classified as stable PD-CN at 18 and 36 months. PD-MCI 2SD was the next most stable group, with $11 \%$ and $12 \%$ being consistently classified as PD-MCI 2 SD at 18 and 36 months, respectively. Nineteen per cent of participants fluctuated over time, with the majority of participants fluctuating between PD-MCI 1SD, 1.5SD and PD-CN.

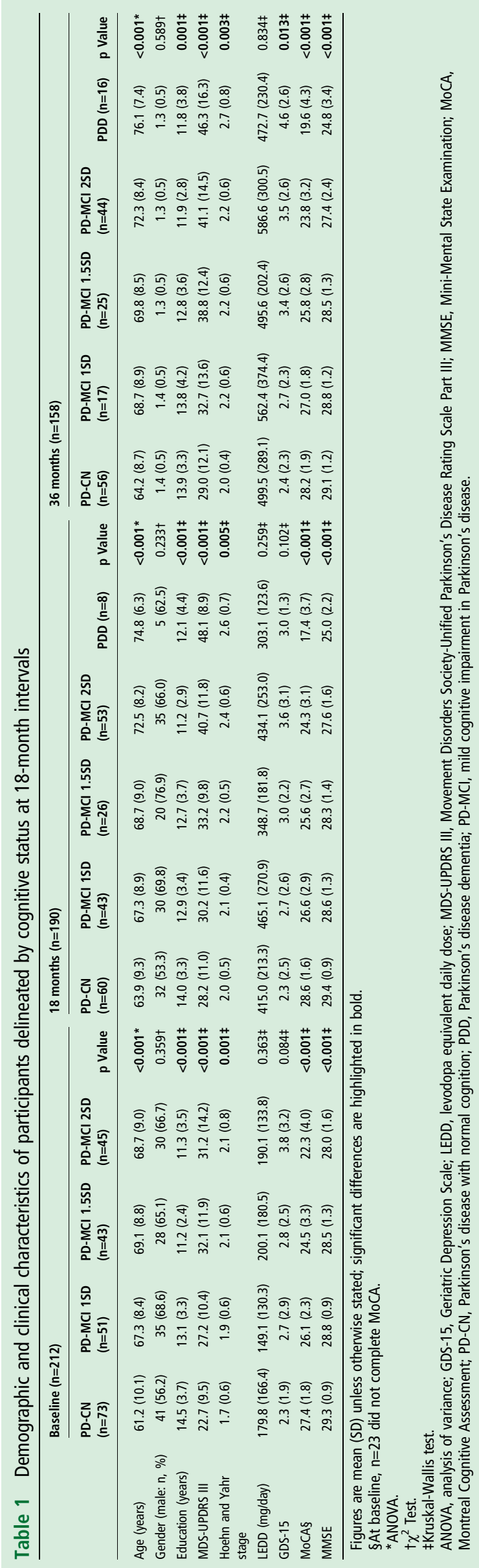



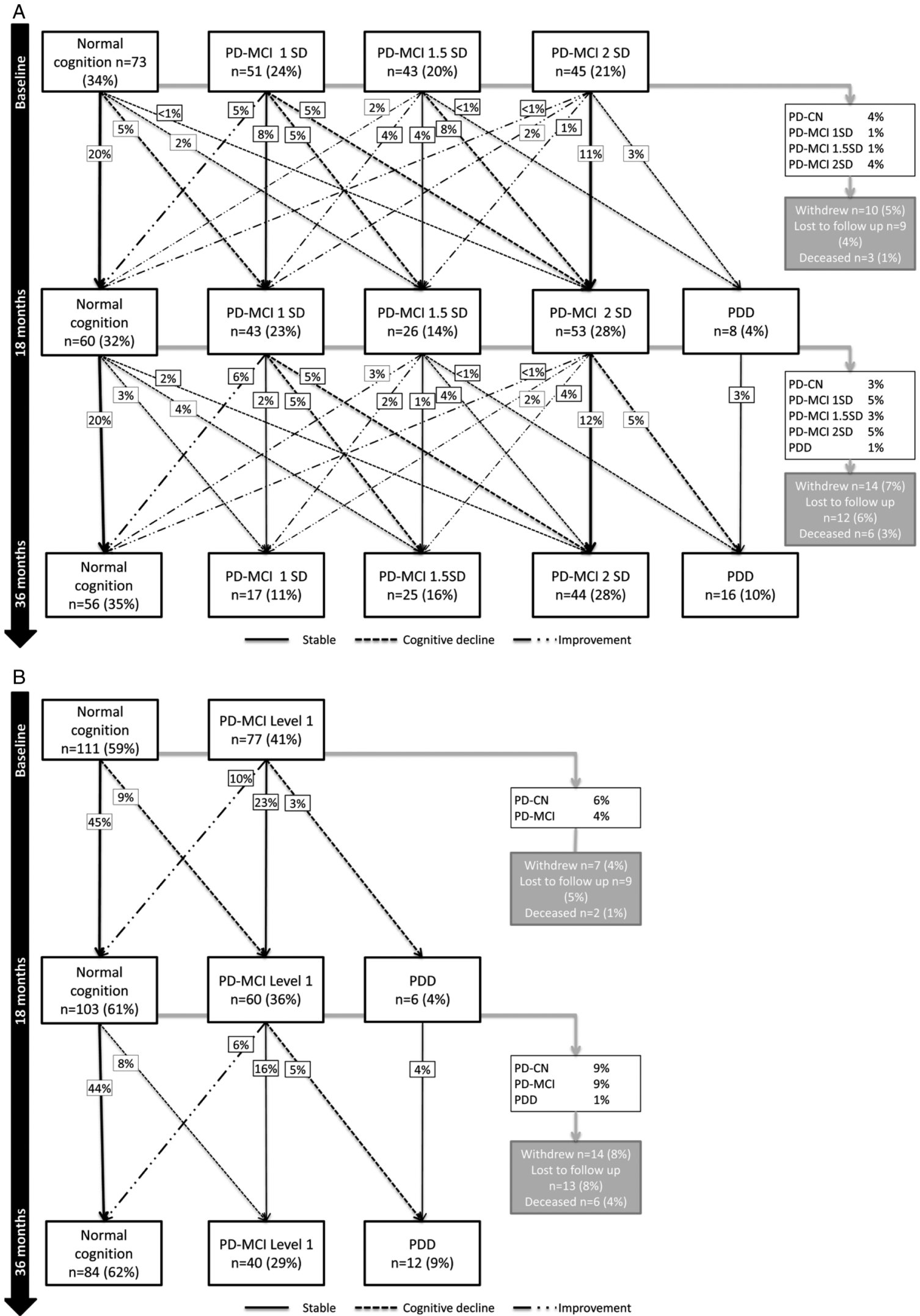

Figure 1 Changes in cognitive classification from baseline to 36 months. (A) Change in cognitive classification using MDS PD-MCI level II criteria to classify PD-MCI using cut-offs of 1, 1.5 and 2SD. (B) Change in cognitive classification using MDS PD-MCI level I criteria to classify PD-MCI using MoCA score. Twenty-three participants did not complete MoCA at baseline and were excluded from this analysis. Percentages relate to the proportion of participants evaluated at that time point: baseline, 18 or 36 months. MDS, Movement Disorders Society; MoCA, Montreal Cognitive Assessment; PD-CN, Parkinson's disease with normal cognition; PD-MCl, mild cognitive impairment in Parkinson's disease; PDD, Parkinson's disease dementia. 
Some participants reverted to PD-CN at 18 (8\%) and 36 months $(10 \%)$ having previously been classified as PD-MCI (cumulatively $18 \%$ ), although this is $<1 \%$ at each time point when applying PC-MCI 2SD criteria. Reverters were significantly younger than non-reverters $(61.3 \pm 9.6$ vs $68.2 \pm 9.2$ years, respectively, $\mathrm{p}<0.01)$ and at baseline had better neuropsychological scores for phonemic and semantic fluency, OTS, PoA, PRM and PAL $(p<0.05$ for all). There were no significant differences between reverters and non-reverters in terms of premorbid IQ, motor severity, LEDD, GDS-15 or anticholinergic use $(p>0.05$ for all).

PD-MCI subtypes were examined. At baseline, 78\% $(n=109)$ were multidomain PD-MCI, while the remaining 22\% $(n=30)$ had single domain impairment. The number of multi domains impaired ranged from two to five; $51 \%$ were impaired in two domains, $25 \%$ in three domains, two participants were impaired in four domains and only one was impaired in all five domains. Executive function was the most commonly impaired domain (59\%), followed by memory (51\%), attention (35\%), language (31\%) and visuospatial function (24\%). Of those diagnosed with PDD, only 3 had single domain PD-MCI at baseline; 16 had impaired executive function at baseline, 12 had impaired visuospatial function, 11 had impaired memory, and 8 had impaired attention while only 5 had impaired language.

Using PD-MCI level I criteria (figure 1B), a greater proportion of participants were classified as PD-CN compared with level II criteria at baseline, 18 and 36 months (59\% vs 34\%, $\chi^{2}=26.7, \mathrm{p}<0.001 ; 61 \%$ vs $32 \%, \chi^{2}=40.1, \mathrm{p}<0.001 ;$ and $62 \%$ vs $35 \%, \chi^{2}=35.4, \mathrm{p}<0.001$, respectively). Between baseline and 18 months, $68 \%$ of participants were stable, $12 \%$ cognitively declined and 10\% improved using PD-MCI level I criteria; 56\% of the remaining participants were stable between 18 and 36 months with $16 \%$ showing cognitive decline and $7 \%$ improvement. Over 36 months, 51\% $(n=95)$ were cognitively stable, $13 \%(n=25)$ cognitively declined and $8 \%(n=15)$ improved.

PD-MCI level I and II criteria classification accuracy was compared using baseline data; PD-MCI 2SD was used to define PD-MCI as it had greater diagnostic stability. In total, 53.2\% $(n=100)$ and $16.0 \%(n=30)$ were identified as PD-CN and PD-MCI, respectively, using both criteria. Data revealed that $5.9 \%(n=11)$ of those defined with level I criteria were falsepositive classifications while $25.0 \%(n=47)$ were false-negative classifications $\left(\chi^{2}=22.5, \mathrm{p}<0.001\right)$. Of those who developed PDD, all participants were identified as PD-MCI using level I criteria and 15 (83\%) were identified using level II criteria with a 2 SD cut-off.

\section{DISCUSSION}

To the best of our knowledge, this is the first study to investigate the stability of PD-MCI using MDS level I and II criteria over time. Using level II criteria, we found that more than a quarter of participants were cognitively stable over 36 months, one-third cognitively declined and 14\% improved. Eight per cent of participants developed PDD, of whom most were previously classified as PD-MCI 2SD. We also found that more than threequarters had multidomain impairment, and $83 \%$ of PDD participants had multidomain impairment at baseline.

Our results show that the operational definition of PD-MCI is an important consideration. Using level I criteria may be more clinically convenient-it is quick to administer and, as our results show, comparably stable. We applied an MoCA score of $<26$ plus subjective cognitive decline to classify PD-MCI, which gave a reasonable indication of patients who had PD-MCI. We did not use the MMSE to classify cognitive impairment as previous studies have suggested that the psychometric properties of the MMSE are not sensitive to PD-MCI. ${ }^{15} 16$

However, one-quarter of patients were subject to type I error using level I criteria compared with using the more comprehensive level II criteria and stricter cut-offs. We used a schedule of 11 cognitive assessments across five cognitive domains with cut-offs of 1, 1.5 and 2SD, below normative values; using the 2SD cut-off gave greater diagnostic certainty. One study with a smaller sample size $(n=76)$ found that using a 1.5SD PD-MCI cut-off, $13 \%$ of participants improved, 3\% fluctuated and 22\% cognitively declined. ${ }^{17}$ In comparison, our data showed that a greater proportion of participants fluctuated (19\%), the majority of whom were classified as PD-MCI using a 1 or $1.5 \mathrm{SD}$ cut-off, whereas a 2SD cut-off was more stable. This could suggest that PD-MCI 2SD has greater diagnostic certainty. A previous study suggested that 2SD below normative values had optimal sensitivity and specificity compared with 1 or $1.5 \mathrm{SD} .^{3}$

We demonstrated that $7-10 \%$ of patients classified as PD-MCI reverted back to normal at 18 and 36 months $(18 \%$ cumulatively), although this rate was much lower using a more conservative 2SD cut-off. A prospective study found a similar conversion rate of MCI to normal cognition (9\%) in participants with persistent PD-MCI at time of diagnosis compared with 3-year follow-up. ${ }^{18}$ Reversions to normal cognition from PD-MCI may be due to a learning effect, an effect of medication or normal fluctuations in cognition. ${ }^{7}$ In a non-PD population, $38 \%$ of people with MCI reverted to normal cognition over a median of 5.1 years; fewer participants reverted if they had amnestic MCI or multidomain MCI and poorer cognitive function. ${ }^{19}$ Poorer cognitive functioning in non-reverters is consistent with the findings of our study; we found that fewer participants reverted if they had multidomain PD-MCI and were impaired in executive function. Roberts et $a l^{19}$ reported that reverters were nearly seven times more likely to later develop MCI or dementia than those with baseline normal cognition, suggesting that $\mathrm{MCI}$ at any time point may have prognostic utility.

The strengths of this prospective study are its longitudinal design, the use of an incident cohort of communityrepresentative patients with $\mathrm{PD}$, and the comprehensive schedule of neuropsychological tests used. As with any longitudinal study, missing data were an issue. This has implications for classification of PD-MCI and could result in a type II error classification of some participants. However, we examined differences between participants at baseline and those with missing data were found to be representative of the whole sample. ${ }^{7}$ A small number of participants did not return for further assessments; these participants may have been pertinent to the findings of this study as they may have had more rapid decline in terms of cognition and disease progression. However, there were no significant differences in baseline scores. Age and education are factors that may affect cognition. We examined the scores and cut-offs for cognitive tests using age and education as covariates. However, remodelling our data did not have a significant impact on PD-MCI classification.

In conclusion, we have shown that PD-MCI is complex and subject to fluctuation over time, which increases diagnostic uncertainty. PD-MCI level I criteria may have greater clinical utility but more comprehensive level II criteria with 2SD cut-offs provide greater prognostic utility. We propose that clinicians could apply level I criteria using an MoCA score of $<26$, which would help to identify PD-MCI, and would have some prognostic value in identifying patients at risk of developing PDD. However, clinicians should be cautious when using the MMSE as scores may not be sensitive to cognitive impairment in PD. 
Acknowledgements In addition to the listed authors, the authors thank the following members of the ICICLE-PD Study Group, all of whom made a significant contribution to the work reported in this paper: Lynn Rochester (Institute of Neuroscience, Newcastle University, UK, principal investigator); Fionnuala Johnston, Claire McDonald and Isobel Sleeman (Institute of Neuroscience, Newcastle University, UK, site investigator); Patrick F Chinnery (Institute of Genetic Medicine, Newcastle University, UK, principal investigator); John T O'Brien (Department of Psychiatry, University of Cambridge, UK, principal investigator); Trevor W Robbins (Department of Psychology, University of Cambridge, UK, principal investigator); Gemma A Cummins and Jonathan Evans (John van Geest Centre for Brain Repair, University of Cambridge, UK, site investigator); David J Brooks (Department of Medicine, Imperial College, London, UK, principal investigator); Keith A Wesnes (Centre for Human Psychopharmacology, Swinburne University, Melbourne, Australia, principal investigator); James B Rowe (Behavioural and Clinical Neuroscience Institute, UK, site investigator).

Contributors RAL was involved with coordination of the study, participant recruitment, data collection, statistical analysis, interpretation of data and drafted the manuscript. AJY, GWD and DPB were also involved with coordination of the study, participant recruitment, clinical assessment, data collection and manuscript revision. TKK was involved with the study design and coordination of the study. He was also involved with participant recruitment, clinical assessment, data collection and manuscript revision. CHW-G was involved with study co-ordination, clinical assessment, data collection and manuscript revision. RAB is a principal investigator and co-applicant for the main funding grant. He was involved with the study design and reviewed the manuscript. DJB is the chief investigator and main applicant for the funding grant. He was involved with the study design, supervised the study and reviewed the manuscript.

Funding This study was funded by Parkinson's UK (J-0802). The research was supported by the Lockhart Parkinson's Disease Research Fund, National Institute for Health Research (NIHR) Newcastle Biomedical Research Unit based at Newcastle upon Tyne Hospitals NHS Foundation Trust and Newcastle University and a NIHR Biomedical Research Centre award to the University of Cambridge/Cambridge University Hospitals NHS Trust.

Disclaimer The views expressed are those of the authors and not necessarily those of the NHS, the NIHR or the Department of Health.

Competing interests None declared.

Ethics approval Newcastle and North Tyneside 1 Research Ethics Committee.

Provenance and peer review Not commissioned; externally peer reviewed.

Open Access This is an Open Access article distributed in accordance with the terms of the Creative Commons Attribution (CC BY 4.0) license, which permits others to distribute, remix, adapt and build upon this work, for commercial use, provided the original work is properly cited. See: http://creativecommons.org/licenses/ by/4.0/

\section{REFERENCES}

1 Hely MA, Reid WG, Adena MA, et al. The Sydney multicenter study of Parkinson's disease: the inevitability of dementia at 20 years. Mov Disord 2008;23:837-44.
2 Litvan I, Goldman JG, Tröster Al, et al. Diagnostic criteria for mild cognitive impairment in Parkinson's disease: Movement Disorder Society Task Force guidelines. Mov Disord 2012;27:349-56.

3 Goldman JG, Holden S, Bernard B, et al. Defining optimal cutoff scores for cognitive impairment using Movement Disorder Society Task Force criteria for mild cognitive impairment in Parkinson's disease. Mov Disord 2013;28:1972-9.

4 Goldman JG, Holden S, Ouyang B, et al. Diagnosing PD-MCl by MDS Task Force criteria: how many and which neuropsychological tests? Mov Disord 2015;30:402-6.

5 Koepsell TD, Gill DP, Chen B. Stability of clinical etiologic diagnosis in dementia and mild cognitive impairment: results from a multicenter longitudinal database. Am J Alzheimers Dis Other Demen 2013;28:750-8.

6 Yarnall AJ, Breen DP, Duncan GW, et al. Characterizing mild cognitive impairment in incident Parkinson disease: the ICICLE-PD study. Neurology 2014;82:308-16.

7 Lawson R, Yarnall A, Duncan G, et al. Cognitive decline and quality of life in incident Parkinson's disease: the role of attention. Parkinsonism Relat Disord 2016;27:47-53.

8 Tomlinson CL, Stowe R, Patel S, et al. Systematic review of levodopa dose equivalency reporting in Parkinson's disease. Mov Disord 2010;25:2649-53.

9 Nicholl CG, Lynch S, Kelly CA, et al. The cognitive drug research computerized assessment system in the evaluation of early dementia-is speed of the essence? Int J Geriatr Psychiatry 1995;10:199-206.

10 Robbins TW, James M, Owen AM, et al. Cambridge Neuropsychological Test Automated Battery (CANTAB): a factor analytic study of a large sample of normal elderly volunteers. Dementia 1994;5:266-81.

11 Tombaugh TN, Kozak J, Rees L. Normative data stratified by age and education for two measures of verbal fluency: FAS and animal naming. Arch Clin Neuropsychol 1999;14:167-77.

12 Ala $T A$, Hughes $L F$, Kyrouac GA, et al. Pentagon copying is more impaired in dementia with Lewy bodies than in Alzheimer's disease. J Neurol Neurosurg Psychiatr 2001;70:483-8.

13 Nasreddine ZS, Phillips NA, Bédirian V, et al. The Montreal Cognitive Assessment, MoCA: a brief screening tool for mild cognitive impairment. J Am Geriatr Soc 2005;53:695-9.

14 Lawson RA, Yarnall AJ, Duncan GW, et al. Quality of life and mild cognitive impairment in early Parkinson's disease: does subtype matter? I Parkinsons Dis 2014:4:331-6.

15 Hoops $S$, Nazem S, Siderowf AD, et al. Validity of the MoCA and MMSE in the detection of $\mathrm{MCl}$ and dementia in Parkinson disease. Neurology 2009;73:1738-45.

16 Burdick DJ, Cholerton B, Watson GS, et al. People with Parkinson's disease and normal MMSE score have a broad range of cognitive performance. Mov Disord 2014;29:1258-64.

17 Santangelo $G$, Vitale $C$, Picillo $M$, et al. Mild cognitive impairment in newly diagnosed Parkinson's disease: a longitudinal prospective study. Parkinsonism Relat Disord 2015;21:1219-26.

18 Pedersen KF, Larsen JP, Tysnes OB, et al. Prognosis of mild cognitive impairment in early Parkinson disease: the Norwegian ParkWest study. JAMA Neurol 2013;70:580-6.

19 Roberts RO, Knopman DS, Mielke MM, et al. Higher risk of progression to dementia in mild cognitive impairment cases who revert to normal. Neurology 2014:82:317-25. 